\title{
Cross-species analysis between the maize smut fungi Ustilago maydis and Sporisorium reilianum highlights the role of transcriptional plasticity of effector orthologs for virulence and disease
}

\author{
Weiliang Zuo ${ }^{1}$, Jasper RL Depotter ${ }^{1, *}$, Deepak K Gupta ${ }^{2,3,4, *}$, Marco Thines ${ }^{2,3,4}$, Gunther \\ Doehlemann ${ }^{1}$ \\ ${ }^{1}$ Institute for Plant Sciences and Cluster of Excellence on Plant Sciences (CEPLAS), University of Cologne, \\ Zuelpicher Str. 47a, 50674 Cologne, Germany; ${ }^{2}$ Department for Biological Sciences, Institute of Ecology, \\ Evolution and Diversity, Goethe University Frankfurt am Main, Frankfurt am Main, Germany; ${ }^{3}$ Senckenberg \\ Biodiversity and Climate Research Centre, Frankfurt am Main, Germany; ${ }^{4}$ Integrative Fungal Research Cluster \\ (IPF), Frankfurt am Main, Germany. \\ *: D. G and J.D contributed equally to this work
}

Author for correspondence: Gunther Doehlemann; email: g.doehlemann@uni-koeln.de

\section{Summary}

- The constitution and regulation of effector repertoires determines and shapes the outcome of the interaction with the host. Ustilago maydis and Sporisorium reilianum are two closely related smut fungi, which both infect maize, but cause distinct disease symptoms. Understanding how effector orthologs are regulated in these two pathogens can therefore provide insights to pathogen evolution and host adaption.

- We tracked the infection progress of $U$. maydis and S. reilianum in maize leaves, characterized two distinct infection stages for cross species RNA-sequencing analysis and identified 207 out of 335 one-to-one effector orthologs being differentially regulated during host colonization, while transcriptional plasticity of the effector orthologs correlated with the distinct disease development strategies.

- By using CRISPR-Cas9 mediated gene conversion, we identified two differentially expressed effector orthologs with conserved function between two pathogens. Thus, differential expression of functionally conserved genes contributes to species specific adaptation and symptom development. Interestingly, another differentially expressed orthogroup (UMAG_05318/Sr1007) showed diverged protein function during speciation, providing a possible case for neofunctionalization.

- Collectively, we showed the diversification of effector genes in related pathogens can be caused both by plasticity on the transcriptional level, as well as through functional diversification of the encoded effector proteins.

Key words: Fungal effectors, cross-species RNA-seq, differentially regulated orthologs, orthogroup, CRISPR-Cas9 gene conversion, Sporisorium reilianum, Ustilago maydis 


\section{Introduction}

During symbiosis, microbes secret effector proteins to facilitate a compatible interaction with their hosts. Effectors hold various functions, such as the suppression of host immunity and host metabolism manipulation to promote host infection. Co-evolution of pathogens with their hosts is driven by an arms race causing selection pressure on perceived effector genes against their recognition by the host immune system (Jones \& Dangl, 2006).

Polymorphisms of effectors are diverse, including presence/absence variation, amino acid substitution, epigenetic modification and transcriptional plasticity, which together determine the outcome of the plant-pathogen interaction (Gijzen et al., 2014; Toruño et al., 2016; Franceschetti et al., 2017; Torres et al., 2020). Until now, many effector studies have focused on genome sequencing and effector prediction and variation amongst phylogenetically related pathogens (Raffaele \& Kamoun, 2012; Sánchez-Vallet et al., 2018); functional elucidation of single effector proteins (Selin et al., 2016); as well as transcriptional changes during host infection in one-to-one host-pathogen interactions (Lanver et al., 2018). However, information available on the transcriptional regulation of effector orthologs across related pathogen species is scarce.

Smut fungi are a group of fungal plant pathogens consisting of more than 1,500 species, which infect mainly grasses including major cereals such as maize, sorghum, wheat, barley and sugar cane (Begerow et al., 2004). A characteristic feature of many smut fungi is their dimorphic growth, where distinct growth states are observed: saprophytic growth as yeast, and filamentous hyphae which initiate the biotrophic interaction with the host (Kämper et al., 2006a). Smuts often infect their host through the root or coleoptile (Martinez et al., 2002; Laurie et al., 2012), from where the fungi proliferate without evident symptoms during the early vegetative growth stage of the host. When plants switch into the reproductive phase, fungal hyphae residing in floral structure may produce massive black/ brown teliospores (the notorious "smut" phenotype). This type of infection style is also found for the smut fungus Sporisorium reilianum, which infects either sorghum ( . $\square$ reilianum $f . s p$. reilianum) or maize (S. $\square$ reilianumf. sp. zeae) and causes head smut disease. Closely related to this is Ustilago maydis, which causes common maize smut disease (Kämper et al., 2006a; Schirawski et al., 2010). While $U$. maydis has the ability to cause disease symptoms on all the aerial maize tissues, $S$. $\square$ reilianum spreads systemically in the plant while it stays close to the vascular bundles and grows up the main stem axis to reach the cob primordia. Ustilago. maydis causes locally restricted plant tumors within a short time of less than 2 weeks under laboratory conditions. Comparative genome analysis of $U$. maydis with $S$. reilianum revealed that the two maize smuts share high genome similarity with regard to gene number and synteny (Schirawski et al., 2010). Furthermore, previous studies suggested that, similar to U. maydis, S. reilianum can efficiently infect maize leaves to spread systemically and cause head smut disease in floral organs. In recent years, several virulence effectors were identified using seedling infection approaches (Schirawski et al., 2010; Ghareeb et al., 2015; 
78

Schweizer et al., 2018). Together, this renders the two closely related species a well-suited model to study regulation and function of orthologous genes.

In $U$. maydis, the expression of effector genes is precisely regulated via a network of hierarchical transcriptional factors (TF), which modulates activity of the transcription of effectors genes at distinct stages of infection (Skibbe et al., 2010; Lanver et al., 2018). The $b$ mating type locus encoded genes, $b E / b W$, form a heterodimer TF, which not only regulates the expression of 38 effectors, but also trigger the expression of several TFs including a $\mathrm{C} 2 \mathrm{H} 2$ zinc finger TF Rbf1 (regulator of bfilament) (Heimel et al., 2010b,a), which then turn on the TF Hdp2 (homeodomain transcription factor 2) and Biz1 ( $b$-dependent zinc finger protein), together activating the expression of effectors important for initialing the biotrophic interaction (Flor-Parra et al., 2006; Heimel et al., 2010b). At later infection stages, the forkhead TF Fox 1 controls effectors necessary for full virulence and inhibition of the host defense after establishment of biotrophic interaction (Zahiri et al., 2010) and the WOPR TF Ros1 (Tollot et al., 2016) regulate effector gene expression related to fungal sporogenesis in mature tumors. A comprehensive set of RNA-seq data covering the whole biotrophic growth phase of $U$. maydis identified distinct expression patterns of effectors and a novel virulence related TF Nlt1 (no leaf tumors1) (Lanver et al., 2018). The expression of effectors in $U$. maydis was also found to be regulated in an organ-specific manner (Skibbe et al., 2010), and a more recent study using RNAsequencing from laser capture micro-dissected cells revealed a host cell-type specific expression pattern for several effector genes (Matei et al., 2018).

Reverse genetics studies identified various $U$. maydis effectors holding distinct virulence functions, and some of these effectors have been functionally characterized on the molecular level (Lanver et al., 2017). Examples are the apoplastic effectors Pit2 and Pep1, which inhibit the maize apoplastic Papain-like cysteine proteases (PLCPs) (Mueller et al., 2013; Misas Villamil et al., 2019) and peroxidase activities (Hemetsberger et al., 2012), respectively. The effectors Cmu1 (Djamei et al., 2011) and Tin2 (Tanaka et al., 2014) are translocated into the host cytoplasm to manipulate host SA and lignin metabolism, respectively. The leaf-specific effector See1 re-activates host DNA synthesis to directly promote tumor formation in bundle sheath cells (Redkar et al., 2015a; Matei et al., 2018). In addition, the secreted repetitive effector protein Rsp3 coats the fungal cell wall and protects it against a host anti-fungal protein (Ma et al., 2018). In contrast, only one S. reilianum specific effector SAD1suppresses the apical dominance of infected maize to increase branch number has been functional characterized in S. reilianum (Ghareeb et al., 2015), and several species-specific and cluster 19A effectors were identified to contribute virulence (Ghareeb et al., 2019). While these studies focused on the effectors' function on the protein level, it remains unknown if and how transcriptional regulation of effectors contributes to virulence and infection style of the pathogen.

Comparative genomic analysis from phylogenetically related plant pathogens suggesting the effector repertories of smut fungi can be classified as core and accessory effector based on the conservation level (Schuster et al., 2018; Beckerson et al., 2019; Depotter \& Doehlemann, 2020; 
115 Depotter et al., 2020). Core effectors are conserved in all phylogenetically related pathogen and may

116 be involved in conserved biological process vital for infection, leading to stabilization of such

117 effectors during speciation (Hemetsberger et al., 2015; Irieda et al., 2019; Thines, 2019).

118 Complementary, accessory effectors are less conserved and only found in few or individual pathogen

119 species and these were supposed to have more subtle/specific functions in virulence. Previous studies

120 suggested that effector orthologs between $U$. maydis and S. reilianum are more functionally conserved

121 compared to Ustilago hordei and/or Melanopsichium pennsylvanicum (Sharma et al., 2014; Redkar et

122 al., 2015b; Stirnberg \& Djamei, 2016).

123 Clustered regularly interspaced short palindromic repeats (CRISPR)-Cas9 was first identified

124 in Streptococcus pyogenes as part of the bacterial immune system against bacteriophage infection

125 (Barrangou et al., 2007), and has been widely adapted as genome editing tool in various organisms

126 including filamentous fungi and oomycetes (Schuster \& Kahmann, 2019). The Cas9 endonuclease

127 generates DNA double-strand break in a specific target that is recognized by the sgRNA (single guide

128 RNA). This double strand break can be repaired by non-homologous end-joining (NHEJ) which

129 eventually results in a gene knock-out mutant. Alternatively, homology direct repair (HDR) with a

130 donor template can be used for the generation of knock-in or gene conversion mutants. In U. maydis,

131 the CRISPR-Cas9 is achieved by expressing the codon-optimized Cas9 protein and sgRNA in an

132 autonomous replication plasmid (Schuster et al., 2016, 2018), which shows high efficiency to generate

133 a selection marker free genome modification mutant. The off-target effects are further reduced by

134 application of high fidelity variant Cas9HF1 (Zuo et al., 2020).

135 In this study, we examined the growth of U. maydis and S. reilianum during leaf infection for

136 recording similar and divergent biotrophic interaction with maize. We investigated the regulation of

137 orthologous genes with an emphasis on effector genes.

139 Materials and Methods

140 Strains, growth conditions and plant infection

141 The mating compatible isolates of $U$. maydis FB1, FB2 and S. reilianum SRZ1, SRZ2 were used for

142 RNA-seq, and the solo-pathogenic U. maydis strain SG200 and its respective knock-out and ortholog 143 conversion mutants were used for virulence tests. All strains were grown in YEPS light liquid medium 144 with $200 \mathrm{rpm}$ shaking or potato dextrose agar (PD, Difco) plate at $28^{\circ} \mathrm{C}$. Escherichia coli strain Top10 145 was used for cloning purpose and grown in dYT liquid medium or YT agar plate with supplement of 146 corresponding antibiotics. Maize variety Early Golden Bantam was used for infection. The plants were 147 grown in a controlled condition of $16 \mathrm{~h} \mathrm{light} \mathrm{at} 28^{\circ} \mathrm{C}$ and $8 \mathrm{~h}$ dark at $22^{\circ} \mathrm{C} .7$ days-old seedlings were 148 infected with a mixture of compatible $U$. maydis/S. reilianum isolates or SG200, and disease 149 symptoms were scored at 12 days after infection.

150

151 Staining and microscopy 
152 For microscopy purposes, $0.1 \%$ tween- 20 was added in the inoculum. To visualize the appressorium,

153 leaves were stained 20 hours post infection with calcofluor solution $(100 \mu \mathrm{g} / \mathrm{ml})$ for $1 \mathrm{~min}$ and briefly

154 rinsed with water (Lanver et al., 2014). To visualize the fungal growth inside the infected leaves,

155 WGA-AF488 (Wheat Germ Agglutinin, Alexa Fluor 488) and Propidium Iodide were used for 156 staining the fungal and plant cell wall, respectively as previously described (Doehlemann et al., 157 2009a). The microscopy was done on a Nikon Eclipse Ti Inverted Microscope with the Nikon NIS-

158 ELEMENTS software (Düsseldorf, Germany) and the photos were taken by HAMAMATSU camera.

159 Confocal microscopy was done by Leica TCS SP8 confocal laser scanning microscope (Leica, Wetzlar,

160 Germany) and the following filters were used excitation $458 \mathrm{~nm}$ and emission 470-490 nm for WGA-

161 AF488 and excitation $561 \mathrm{~nm}$ and emission 590-603 $\mathrm{nm}$ for Propidium Iodide.

162

\section{RNA preparation and RNA-seq}

164 For RNA preparation, 2-cm long of $3^{\text {rd }}$ leaf sections from more than 15 individual plants were 165 collected for each sample. The compatible haploid U. maydis and S. reilianum cells from cultures with $166 \mathrm{OD}_{600}$ around 0.8 were spun down and mixed in a 1:1 ratio as axenic culture (AC) control. The plant 167 tissues and cell pellets were ground into fine powder with liquid nitrogen and the RNAs were prepared 168 using TRizol (Thermo Fisher, Waltham, USA) according to the manufacture's protocol and followed 169 by DnaseI digestion (Thermo Fisher, Waltham, USA). The RNA libraries were prepared using an 170 Illumina TruSeq Stranded mRNA kit (Illumina, San Diego, USA), and paired-end sequencing was 171 performed on the a HiSeq4000 platform to produce $2 \times 75$ bp long reads at the Cologne Center for 172 Genomics (Cologne, Germany). Three independent biological replicates have been sampled and used 173 for RNA-seq analysis.

174

\section{Data analysis}

176 For the gene expression analysis of U. maydis and S. reilianum individually, reads of three biological 177 replicates were filtered using the Trinity software (v2.9.1) option trimmomatic under the standard 178 settings (Grabherr et al., 2013). They were mapped to a reference assembly using Bowtie 2 (v2.3.5.1) 179 with the first 15 nucleotides on the 5'-end of the reads being trimmed (Langmead \& Salzberg, 2012). 180 The reference genome was either the genome assembly of $U$. maydis (Kämper et al., 2006b) or $S$. 181 reilianum (Schirawski et al., 2010) combined with that of Z. mays B73 version 3 (Schnable et al., 182 2009). Reads were counted to the U. maydis and Z. mays loci using the R package Rsubread (v1.34.7) 183 (Liao et al., 2019). edgeR package v3.26.8 was used for statistical analysis of differential gene 184 expression and pairwise comparison was conducted using Generalized linear models (glm) (Robinson 185 et al., 2009). Genes with $\log 2$ fold change $>1$ and $p<0.05$ were considered as differentially regulated 186 between timepoints, and genes with $\log 2$ fold change $<1, p<0.05$ or $\log 2$ fold change $>1$ but $p>0.05$ 187 were considered as not significantly differenr in the induced pattern analysis. The induced patterns of 188 effectors were defined based on the differentially regulation relationships between AC, 2 dpi and 4 dpi 
189 samples with following criterias: pattern1 are genes induced in planta (expression levels at 2 dpi and 4

190 dpi were significantly higher than AC samples) but the 4 dpi expression level is higher than 2 dpi;

191 genes classified as pattern 2 were induced in planta, but the expression levels were similar between 2

192 and 4 dpi; pattern 3 genes were only induced at 2 dpi and had dropped again at 4 dpi to AC level;

193 pattern 4 genes were significantly induced in planta at both timepoints, but the expression level of 2

194 dpi is higher than 4 dpi; pattern 5 genes are only induced at 4 dpi.

195 A phylogenetic tree was constructed including the smut species U. hordei (Laurie et al., 2012), M.

196 pennsylvanicum (Sharma et al., 2014), S. scitamineum (Taniguti et al., 2015), S. reilianum (Schirawski

197 et al., 2010) and U. maydis (Kämper et al., 2006a). Moesziomyces antarcticus was used as an

198 outgroup (Morita et al., 2013). A phylogenetic tree was constructed based on 1643 Benchmarking

199 Universal Single-Copy Orthologs (BUSCOs) from the database "basidiomycota_odb10" that were

200 present in single copy in all members investigated in the phylogenetic reconstruction (Seppey et al.,

201 2019). For every gene, orthologs were aligned using MAFFT (v7.464) option "--auto" (Katoh \&

202 Standley, 2013). These aligned gene sequences were then concatenated for every species and used for

203 tree construction using RAxML (v8.2.11) with the substitution model "GTRGAMMA" and 100

204 bootstraps (Stamatakis, 2014).

205 For comparison of ortholog expression, high quality paired end transcriptomic reads were mapped 206 using Bowtie2 with default parameters over all orthologous genes of Ustilago maydis and Sporisorium 207 reilianum as calculated using OrthoMCL. Average coverage of mapping of each gene was calculated 208 from the sorted BAM file by summing the coverage of each base of a gene and then dividing this by 209 the length of that gene. The relative expression value of a gene was calculated by using the following 210 formula : Relative Expression of Gene $(X)=\frac{\frac{\sum_{1}^{n} \operatorname{Coverage}(X) \times(N-n)}{n}}{(M-m) /(N-n)}$, Where $\mathrm{n}=$ Length of the gene

211 (X), $\mathrm{N}=$ Total number of bases in all genes ; $\mathrm{m}=$ Number of bases mapped to gene $(\mathrm{X}) ; \mathrm{M}=$

212 Number of bases mapped to all genes. Student t-test and/or one sample t-test were used for 213 significance test with Benjamini-Hochberg $p$ value correction for multiple comparison. Orthologs with 214 fold change $>3$ and adjust $p<0.05$ were considered as differentially regulated ortholog between $U$. 215 maydis and $S$. reilianum.

216

217 Gene ontology and GO enrichment analysis

218 The gene ontology classification and overrepresentation analysis were done by using the U. maydis 219 annotation data from PANTHER (pantherdb.org). The overrepresentation was conducted by using 220 Fisher's exact test with Bonferroni correction.

221

\section{Quantitative Real-Time PCR}

223 For biomass quantification, the DNA of infected leaves from three biological replicates was prepared 224 by Buffer A (0.1M Tris-HCl, 0.05M EDTA, $0.5 \mathrm{M} \mathrm{NaCl}, 1.5 \%$ SDS $)$, then further purified by 

ng of DNA was used for $\mathrm{qPCR}$, and $2^{-\Delta \mathrm{Ct}}$ was calculated to determine the ratio between fungal peptidylprolyl isomerase (ppi) and maize GAPDH.

228 For gene expression, the total RNA was reverse-transcribed with Oligo(dT) primer by using RevertAid First Strand cDNA Synthesis Kit (Thermo Scientific, Waltham, USA), and the expression level of each effectors were determined by $2^{-\Delta \mathrm{Ct}}$ ratio between effector genes and ppi genes from respective species. The qPCR was conducted in CFX96 Real-Time PCR Detection System (Bio-Rad, Hercules, USA) with GoTaq qPCR mix (Promega, Madison, USA). The primers designed in this study were listed in Table. S1.

234

\section{Gene conversion in $\boldsymbol{U}$. maydis by CRISPR and disease scoring}

CRISPR-Cas9 mediated genome editing was used to generate the effector ortholog conversion mutants in $U$. maydis solo-pathogenic strain SG200. An sgRNA was designed to target the coding region or the promoter region of the effector to generate the double strand break, which was then repaired by homology-directed repair using a donor plasmid as template. The design of sgRNA and cloning was done as previously described (Zuo et al., 2020). To construct donor plasmid, $1 \mathrm{~kb}$ fragments flanking the effector coding region or promoter regions and the respective $S$. reilianum ortholog open reading frame or promoters were amplified by Phusion DNA polymerase (NEB, Ipswich, USA) with primers (Sigma Aldrich, St. Louis, US) listed in Table. S1 and cloned into pAGM1311 vector by Gibson assembly (New England Biolabs, Ipswich, USA). The CRISPR plasmid and circular donor plasmid were co-transformed into SG200 protoplast and mutants were singled out on PD plate with $2 \mu \mathrm{g} / \mathrm{ml}$ carboxin for CRISPR plasmid first then transfer to PD plate without antibiotic to get rid of CRISPR plasmid. The resulting conversion mutants have no antibiotic resistance. Correct integration of the recombinant DNA was confirmed by southern blot (not shown). The conversion mutants and corresponding effector deletion mutants (Schilling et al., 2014) were used for infection. Disease scoring was done as previous described at $12 \mathrm{dpi}$. Disease indexes 9, 7, 5, 3, 1 and 0 were assigned to dead, heavy tumor, tumor, small tumor, chlorosis and normal symptom, respectively. The number of diseased plants were multiplied by the corresponding disease index, and the sum was divided by the total number of plants used for infection to give an average disease index. Student $t$-test was used for significance test of disease index from three biological replications.

\section{Results}

\section{Maize leaf infection of $S$. reilianum and $U$. maydis}

258 For a comparative transcriptomics approach between $S$. reilianum and $U$. maydis, we first tracked

259 infection and fungal growth inside the maize leaves to record the milestone events during infection 260 and define appropriate timepoints for analysis (Fig. 1). 18-24 hours post infection, the compatible 261 sporidia cells of both pathogens, mated on the leaf surface, and dikaryotic hyphae formed appressoria 
262 to penetrate the leaf surface (Fig. 1a). At 2 days post infection (dpi), all infected leaves showed 263 chlorosis as a first visible indication of successful infection (Fig. S1a). Microscopic analysis of WGA264 AF488 stained hyphae showed similar colonization in the leaf vascular tissue for both smut fungi (Fig. 265 1b). At 3 dpi, the earliest microscopic difference was observed between S. reilianum and U. maydis: in 266 alignment to our previous report (Matei et al., 2018), host bundle sheath cells underwent de novo cell 267 division in $U$. maydis infected leaves, indicating the initiation of tumorigenesis. In contrast, $S$. 268 reilianum further accumulated in the leaf vein and showed aggregation as a string of round cells (Fig. 269 1c). At 4 dpi, the first visible small tumor structures can be observed on $U$. maydis infected leaves, 270 while S. reilianum infected leaves showed individual events of necrosis (Fig. S1a) and more 271 frequently cell aggregation (Fig. 1d, Fig. S1b). In parallel to the microscopic observation, fungal 272 biomass in the infected leaves was quantified for both pathogens. The relative amount of both fungal 273 pathogens increased similarly during infection until 3 dpi. Interestingly, at 4 dpi S. reilianum showed a 274 significantly higher abundance compared to $U$. maydis, as the relative amount of S. reilianum doubled 275 while the relative amount of $U$. maydis was maintained in comparison to 3 dpi (Fig. 1e).

\section{Expression profiling of $\boldsymbol{U}$. maydis and $S$. reilianum during maize leaf colonization}

278 Based on our microscopic and biomass analysis, we decided to collect samples at 2 dpi to represent an infection stage where both $U$. maydis and S. reilianum successfully establish the biotrophic interaction with the host but do not show detectable differences in microscopical growth and biomass accumulation. Samples were also taken at 4 dpi when $U$. maydis tumorigenesis is initiated, while $S$. reilianum exhibits extensive tissue colonization without inducing morphological changes in the leaf. As control samples, cell pellets from axenic culture (AC) of both pathogens were used for RNA extraction. All samples have been generated in three independent biological replicates. In total, more than $670 \mathrm{M}$ paired-end reads were generated, of which over $93.7 \%$ of reads were uniquely mapped to either $U$. maydis, S. reilianum or the maize genome, respectively. The changes of fungal transcripts in the RNA-seq samples confirmed the biomass quantification results by qPCR. At 2 dpi, around $2 \%$ of fungal transcripts were mapped from both pathogens, and at $4 \mathrm{dpi}$, S. reilianum reads increased to $12.5 \%$ in the infected samples, which is significantly higher compared to U. maydis infected tissues $(7.5 \%)$ (Fig. 1f). Principal component analysis (PCA) analysis of U. maydis and S. reilianum samples showed that three biological replications from different conditions form distinct clusters, and variations between different conditions were larger than the variation of biological replications within the same condition (Fig. 2a). To further analyze the RNA-seq data, we normalized reads counts and investigated the differentially expressed genes (DEGs) by edgeR (Robinson et al., 2009). After filtering the low- or not-expressed genes between samples, 6537 out of 6765 U. maydis genes and 6456 out of 6673 S. reilianum genes were used to identify DEGs. Compared to axenic culture, dramatic changes in gene expression were observed during seedling infection. Around 19.3-24.2\% 
12.1-19.1\% genes were down-regulated (Fig. 2b). We detected 435 out of 467 effector genes from $U$.

300 maydis and 454 out of 489 from S. reilianum were expressed. As expected, most effector genes were

301 specifically expressed in the biotrophic growth stage (311 in U. maydis and 307 in S. reilianum,

302 respectively) and showed higher degrees of transcriptional induction compared to non-effector genes

303 (Fig. 2c, d), which reflects the explicit role of effectors in host infection. Three biological replicates of

304 quantitative RT-PCR were conducted to confirmed the expression of several effector genes including

305 infection markers such as Pit2 and Pep1 from our RNA-seq data (Fig. S2a, b).

306 We clustered effector genes into all five possible expression patterns between three conditions (axenic

307 culture, 2 dpi, 4 dpi) (Fig. 3a). This classified groups of effector genes (i) showing constitutively

308 increasing induction (pattern 1), (ii) induced in-planta on a stable level (pattern 2), (iii) being only

309 transiently expressed (pattern3), (iv) dropping at 4 dpi (pattern 4), (v) or only being induced at 4 dpi

310 (pattern 5). In U. maydis and S. reilianum, 227 effectors from each species were highly expressed

311 across the whole biotrophic growth phases (pattern 1, 2 and 4), while only 14 U. maydis effectors and

3128 S. reilianum effectors were transient induced at 2 dpi (pattern 3) and similar number of effectors

313 were only required in the 4 dpi (23 from U. maydis vs. 25 from S. reilianum as pattern 5) (Fig. 3b).

314 The expression patterns are found to reflect previously observed virulence function of known effector

315 genes. For example, the core effector UmPep1(UMAG_01987) (Doehlemann et al., 2009b;

316 Hemetsberger et al., 2015) was stably expressed and clustered as pattern 2, while genes coding for the

317 known virulence factors UmPit2 (UMAG_01375) (Misas Villamil et al., 2019),

318 UmCmul(UMAG_05731) (Djamei et al., 2011), UmTin2 (UMAG_05302) (Tanaka et al., 2014) and

319 UmRsp3 (UMAG_03274) (Ma et al., 2018) group to pattern 1 cluster. Interestingly, S. reilianum

320 orthologs of these characterized effectors displayed different induction patterns, keeping a steady level

321 between 2 and 4 dpi. In particular, we identified 100 out 467 of $U$. maydis effectors being induced in

322 pattern 1, which is significantly higher compared to $S$. reilianum (43/488), whereas 184 out of $488 S$.

323 reilianum effector genes were showing stable expressing levels, which is higher as compared to $U$.

324 maydis (136/467) (Fig. 3b). This enrichment of different induction patterns of effector genes

325 suggested different requirements in disease for the two species.

326

\section{Differential regulation of effector orthologues in $\boldsymbol{U}$. maydis and S. reilianum}

328 To better understand how U. maydis and S. reilianum orchestrate their respective effector repertoires

329 with respect to their differential disease development, we directly compared the relative expression

330 levels of effector orthologs. We used orthoMCL to identify one-to-one ortholog pairs between $U$.

331 maydis and S. reilianum and obtained 6005 one-to-one ortholog pairs including 335 effectors. This

332 means that for avoiding ambiguous results due to functional redundancy or paralog compensation,

333 one-to-many or many-to-many ortholog pairs were not included in this analysis. Relative expression

334 was corrected for putative differences in length between the orthologs. PCA analysis based on the

335 relative expression of 6005 ortholog pairs confirmed that plant associated samples form distinct 
336 clusters on time and species levels. In contrast, the axenic cultured samples of the different species

337 have minor differences and clustered together (Fig. 4a). Pearson correlation analysis displayed similar

338 results and suggested the difference between samples increased at 4 dpi compared to 2 dpi, as the 339 correlation decreased (Fig. S3a).

340 Next, we compared the relative ortholog expression levels to identify differentially expressed 341 orthologs (DEOs) in the two smut fungi. In total, we found 769 DEOs with comparatively higher 342 expression in U. maydis and 737 DEOs being more highly expressed in S. reilianum, respectively (Fig. 343 4b). The largest number of DEOs (835) was detected from axenic cultured samples (Fig. 4b), and 344 around 30 and $50 \%$ of these DEOs from $U$. maydis and S. reilianum were only confined in this stage 345 (Fig. S3b). The DEO number dropped to 323 at 2 dpi when the pathogens had switched from in-vitro 346 yeast growth to the initial filamentous, biotrophic growth in the host tissue. At 4 dpi the number 347 increased to 747, which likely reflects the significant differences in disease development between the 348 two pathogens, i.e. initiation of tumorigenesis by $U$. maydis versus extended proliferation of $S$. 349 reilianum for systemic spreading. We also detected more U. maydis-induced DEOs during infection, 350 particularly at $2 \mathrm{dpi}$, when 227 DEOs were $U$. maydis-induced but only 97 DEOs for $S$. reilianum (Fig. 351 4b). However, we could not detect overrepresented gene ontology terms, except for 4 dpi, where an 352 enrichment of cellular lipid catabolic process (GO:0044242) was detected in the higher expressed $U$. 353 maydis DEOs. The DEOs were involved in different biological processes related to pathogen growth 354 (Fig. 4c), suggesting that during the yeast and biotrophic growth no particular cellular or metabolic 355 pathway was selected to promote cell growth in either $U$. maydis or S. reilianum.

356 From the 335 one-to-one effector orthologs, more than $60 \%$ were differentially regulated, including 357100 U. maydis- and 102 S. reilianum-highly expressed effectors, from which 32 and 35 showed 358 consistently enhanced expression during the two biotrophic time points (Fig. 4d). The number of 359 effector DEOs also increased from 99 at 2 dpi to 170 at 4 dpi as disease developed. Previously 360 identified pathogenesis associated TFs might be responsible in the regulation of effector DEOs, 361 particularly at $4 \mathrm{dpi}$, since differential expression was found for virulence related TFs Rbfl, Bizl, $362 H d p 2$, Fox 1 and Nlt1 (Fig. S4). TF Rbfl was highly induced in S. reilianum, especially at 4 dpi, the 363 transcript levels were 17-fold higher than those in U. maydis, which may lead to the high abundance of 364 Biz1 in S. reilianum (Fig. S4). On the other hand, the expression level of TFs Hdp2, Fox1 and Nlt1 365 were significantly higher in U. maydis at $4 \mathrm{dpi}$, which could indicate that these TFs play a role in the 366 regulation of effectors highly expressed in U. maydis during tumorigenesis (Fig. S4). Besides these, 367 the TF ROS1 showed similar expression level in both species, which may reflect its dedicated function 368 in sporogenesis, a process which needs to be initiated in both pathogens for completing the pathogenic 369 like cycle (Fig. S4). The expression of ROS1 is dramatically increased since 6 dpi in U. maydis that is 370 corresponding to the initiation of sporogenesis (Tollot et al., 2016), which may explain why we did 371 not observe its differential expression between two species. Cluster 19A (Fig. S5a) is the largest 372 effector cluster, which is a crucial determinant of virulence in both two pathogens (Brefort et al., 2014; 
373 Ghareeb et al., 2019). We observed that 10 out of 14 effectors residing in cluster 19A were U. maydis-

374 enhanced, whereas only two were highly expressed in S. reilianum, including the neofunctionalized

375 Tin2 (Tanaka et al., 2019) (Fig. S5b).

376 In a previous study, Schuster et al. analyzed 12 smut related basidiomycete and suggested that effector

377 repertoires of smut fungi comprise sets of core and accessory effectors (Schuster et al., 2018). Based

378 on this information, we classified the 335 one-to one effector pairs into plant smut core effectors,

379 which were present in all 4 monocot smut fungi, including Ustilago hordei and Sporisorium

380 scitamineum, and accessory effectors, which present in U. maydis and S. reilianum but not in all other

381 plant smut fungi (Fig. 4e). Melanopsychium pennsylvanicum was included in this analysis due to its

382 close phylogenetic relationship to monocot smut pathogens, which suggests a recent host jump event

383 (Sharma et al. 2014). In total, we identified 191 core effectors and 144 accessory effectors. At 2 dpi,

38440 core effectors and 31 accessory effectors were differentially expressed between $U$. maydis and $S$.

385 reilianum, and these numbers were significantly increased to 67 and 61 at 4 dpi, respectively (Fig. 4f).

386 We did not find accessory effectors subjected to more intensive transcription regulation compared to

387 core effectors (Fig. 4f), suggesting that differential regulation of both core and accessory effectors is

388 needed for the distinct pathogenic development in the two pathogens.

389

390 Functional conservation and neofunctionalization of differentially expressed effector orthologs

391 Several U. maydis effectors have been functionally characterized, which provides a reference to link 392 the ortholog expression with their biological functions. The conserved core effector Pep1 393 (Hemetsberger et al., 2012, 2015; Sharma et al. 2019), as well as the virulence factors Pit2 (Misas 394 Villamil et al., 2019) and Cmu1 (Djamei et al., 2011) were highly induced during biotrophic growth 395 (Table. S2) and showed similar expression level between U. maydis and S. reilianum, which is 396 consistent with their crucial roles in maize immunity inhibition (Fig. 5a). The effectors Rsp3 (Ma et 397 al., 2018) and ApB73 (Stirnberg \& Djamei, 2016) had been validated in previous studies for their 398 conserved function between two smut fungi by ortholog complement assay, and also these genes show 399 comparable expression levels (Fig. 5a). Also the See1 effector, which in U. maydis has a virulence 400 function specifically for leaf tumor-formation (Redkar et al., 2015a), is expressed in S. reilianum both 401 at 2 and 4 dpi (Fig. 5a). Another effector DEO which caught our attention is Tin2. SrTin2 402 neofunctionlized during speciation between U. maydis and S. reilianum (Tanaka et al., 2019). We 403 found a significantly enhanced expression of SrTin2 compared to UmTin2 (Fig. 5a), in line with the 404 neofunctionalisation, as the transcriptional level divergence could be involved to promote the 405 functional divergence.

406 From a set of 20 organ-specific effectors, we found 9 effector DEOs and 8 of them were leaf-specific 407 effectors, from which 4 were validated as virulence factors from a deletion mutant screen (Schilling et 408 al., 2014) (Fig. 5a). Orthogroups UMAG_11060/Sr14941, UMAG_05306/Sr10059 and 409 UMAG_05318/Sr10075 were particularly interesting as they were induced in both pathogens (Table. 
410 S2), however, the transcripts levels of the orthologs in U. maydis were at least 4.8 folds (for 411 orthogroup UMAG_11060/Sr14941 at 2 dpi) to more than 50 times (orthogroup 412 UMAG_05318/Sr10075 at 4 dpi) higher compared to their S. reilianum orthologs (Table. S2). These 413 effectors are specifically required for $U$. maydis induced leaf tumors (Schilling et al., 2014). Thus, 414 their low expression in S. reilianum might suggest that these genes are of lesser importance for the 415 basal establishment of biotrophic growth. We assessed the protein function of these DEOs to test if 416 functional diversification occurred and is associated with differentially expression, as in the case of 417 Tin2. We converted the open reading frame of the candidate effectors in U. maydis to their respective 418 S. reilianum orthologs. For the generation of in situ seamless mutants we used a CRISPR-Cas9 419 approach that allowed marker-free selection of gene-replacement mutants in U. maydis (Fig. 5b). In 420 each case, an sgRNA was designed to target the effector gene to induce a double strand break, which was then repaired by HDR with a donor plasmid containing the $S$. reilianum effector ortholog, flanked by $1 \mathrm{~kb}$ homologs arms (Fig. 5b). This CRISPR-Cas9 mediated gene conversion eliminated potential ectopic effects and allowed expression of the orthologs under the native in-situ cis- or trans- regulatory elements.

In maize infection assays, conversion of UMAG_05306 and UMAG_11060 to their S. reilianum orthologs did not affect the virulence compared to the $U$. maydis progenitor strain SG200, while the deletion of these two effectors resulted in significantly reduced virulence (Fig. 5d). However, conversion of UMAG_05318 into its S. reilianum ortholog Sr10075 significantly reduced virulence to the similar level of the deletion mutant, which indicates that Sr10075 might have a different function, or lost its activity during speciation (Fig. 5d). In S. reilianum, Sr10075 has a paralog Sr10079, which has only low sequence identity to UMAG_05318 and therefore was not detected in our one-to-one ortholog analysis (Fig. S6a, b). Deletion of Sr10079 was previously shown to reduce the virulence in $S$. reilianum (Ghareeb et al., 2019). Conversion of UMAG_05318 into Sr10079 resembled virulence defect of the UMAG_05318 deletion mutant. Thus, neither Sr10075, nor Sr10079 can functionally replace UMAG_05138, suggesting the functional divergence of the orthogroup UMAG_05318/Sr10075 - Sr10079 during speciation, which identifies them as an interesting objective to test for possible neofunctionalization.

438 To further confirm and investigate how promoters regulate the expression of DEOs, we used the 439 CRIPSR-Cas9 mediated conversion approach to replace the promoter of the $U$. maydis induced DEOs 440 UMAG_11060 and UMAG_05306 with their respective S. reilianum counterparts in SG200 (Fig. S7a), 441 and quantitative RT-PCR was used to detect the chimeric gene expression. Infected samples at the time points when the differential expression levels were detected in RNA-seq ( 2 dpi for $U M A G \_11060$ and 4 dpi for $U M A G \_05306$, Fig. 5a) were tested. Expressing $U M A G_{-} 11060$ under Pro ${ }^{\text {sr14941 }}$ showed

444 lower expression at 2 dpi (Fig. S7b), which was similar to our RNA-seq analysis, indicating the cis445 regulation in the promoter region determined their differential expression. However, Pro ${ }^{\text {Sr10059 }}$ 446 surprisingly drove a similar expression level of UMAG_05306 at 4 dpi in $U$. maydis genetic 
447 background, suggesting a trans regulator or distal enhancer, which is variable between two species

448 control the differential expression (Fig. S7b).

449 Together, the results of the gene conversion approach showed that functional diversification of

450 effector orthologs can be caused on two levels: (i) the differential expression of orthologous genes

451 which encode functionally conserved proteins (as found for orthogroups UMAG_05306/Sr10059 and

452 UMAG_11060/Sr14941), (ii) the functional divergency of effectors on the protein level, which further

453 fine-tuned on transcription levels for functional adaption (iii) the regulations of DEO is complicated,

454 involving both cis- and trans-regulatory elements.

455

\section{Discussion}

457 In this study we applied a cross-species transcriptome comparison between $U$. maydis and S. reilianum

458 to investigate transcriptomic changes of effector orthologs during maize leaf infection.

459 A crucial challenge in cross-species RNA-seq is to determine the right timing for sampling to exclude

460 that the observed differences result rather from the different disease progression states than from a

461 differential regulation as such. Sucher et al. (2020) collected samples from the edge of disease lesions,

462 which were smaller than $25 \mathrm{~mm}$ in diameter, as indication of comparable infection stage to study the

463 different host responses to Sclerotinia sclerotiorum for quantitative disease resistance (Sucher et al.,

464 2020). Time resolved microscopes were applied in Fusarium virguliforme to facilitate RNA-seq to

465 study its transcription plasticity on different hosts (Baetsen-Young et al., 2020). In our experiment, we

466 conducted a series of microscopy combined with qPCR-based biomass quantification, and selected

467 two biotrophic interaction phases representing a comparable ( $2 \mathrm{dpi}$ ) and a distinct colonization stage (4

468 dpi) for RNA-seq.

469 When comparing the induction profiles of effectors in U. maydis and S. reilianum respectively, we

470 found a significantly higher number of S. reilianum effectors showing stable expression between 2 and

$4714 \mathrm{dpi}$, while in U. maydis more effectors displayed an increasing expression over time. We further

472 focused on the expression regulations of one-to-one effector orthogroups. Overall, 207 out of 335 one-

473 to-one effector orthologs were differentially expressed between $U$. maydis and $S$. reilianum during

474 biotrophic interaction, and the number of effector DEOs increased at 4 dpi. The difference in effector

475 induction patterns and differential regulation of effector DEOs are consistent with the appearance of

476 different symptoms. At $4 \mathrm{dpi}, U$. maydis triggers local host cell proliferation to produce tumors, which

477 will require the high transcript abundance of effector genes related to tumorigenesis; in contrast, $S$.

478 reilianum sustaining stable expression levels of effectors likely reflects maintenance of the biotrophic

479 interaction in already colonized tissue and continuation of systemic proliferation through the leaf veins

480 with inducing structural changes in the host tissue

481 Not only effector genes, but also pathogenesis related transcription factors were found to be

482 differentially regulated, which in turn is probably playing a crucial role in the control of effector DEO

483 expression. TFs Rbf1 and Biz1 were highly expressed in S. reilianum, which may be responsible for 
484 the expression of $S$. reilianum enriched effector DEOs. However, the abundance of TF Hdp2 485 transcripts, which is activated by Rbf1 (Heimel et al., 2010b), was more highly expressed in U. maydis, 486 indicating the complicated regulation of TF cascade. The promoter conversion of Pro ${ }^{\text {Sr10059 }}$ showed 487 comparable expression with Pro ${ }^{\mathrm{UMAG} \_05306}$ in U. maydis (Supplementary Figure S7), indicating the potential trans effects such as transcription factors or distal enhancers might be driving differential regulation while for orthogroup UMAG_11060/sr14941, cis-regulatory elements in promoter region likely determines the expression pattern. Until now, only few direct target genes of TFs were identified by CHIP-seq in smut fungi (Tollot et al., 2016). Elucidation of the binding motif of these pathogenesis related TFs and combined with the promoter analysis of effector DEOs, which were obtained in this study could be a starting point for a more detailed study on the specific roles of TFs in the orchestration of effector expression with respect to the adaptation to different infection styles in pathogen speciation. A major conclusion which can be drawn from the comparative transcriptome analysis is that the expression levels of effector orthologs between $U$. maydis and $S$. reilianum are related to their function: effectors with general virulence functions showed similar expression levels between both smut fungi, whereas effectors adapted to execute specific processes such as the leaf-tumor specific effectors of $U$. maydis showed significant differences in their transcriptional regulation. UMAG_05306 and UMAG_11060, whose disruption resulted in reduced virulence in $U$. maydis and were functionally conserved with respect to the corresponding S. reilianum orthologs. This implies that the functional diversification of effectors can be driven on the transcriptional level and is not necessarily linked to a changed protein function. However, UMAG_05318 provides another example for combination of transcriptional plasticity and functional diversification of the protein level. While the orthogroups UMAG_05318/Sr10075 or Sr10079 and Umtin2/Srtin2 were expressed during leaf infection both in $U$. maydis and $S$. reilianum, they also show similar induction pattern during biotrophic interaction. However, when comparing the expression levels of the orthologs, UMAG_05318 is more than 50 times higher than its $S$. reilianum ortholog, while Srtin2 has a 10 times higher expression compared to its respective ortholog in $U$. maydis. Until now, the molecular mechanisms of these differentially regulated organ specific effectors are not elucidated in $U$. maydis, and whether they also contribute to

512 virulence in S. reilianum is largely unknown. Deletion of Sr10075 alone did not seem affect virulence,

513 while a knock-out Sr10079 reduced S. reilianum virulence (Ghareeb et al., 2019). The orthologs of 514 UMAG_05318 were found in S. scitamineum and S. reilianum f. sp. reilianum, but not in U. hordei 515 and M. pennsylvanicum. Elucidation of the function of this ortholog group and its role in smut fungi 516 evolution and speciation will be our next aim. Interestingly, both effectors showing functional 517 divergence are located in effector cluster 19A, which is the largest virulence cluster in both pathogens 518 (Brefort et al., 2014; Ghareeb et al., 2019) and several U. maydis highly expressed effector DEOs 519 were also identified in this cluster from our study. However, the impact of 19A deletion on $S$. 520 reilianum virulence was monitored by the head smut phenotype in the maize inflorescence (Ghareeb et 
521 al., 2019). Thus, it could be possible that these effectors were highly expressed in a different 522 time/spatial pattern to contribute to the systemic spreading of S. reilianum. On the other hand, it would 523 be tempting to study if higher expression of cluster 19A effectors could promote the adaptation of $S$. 524 reilianum to leaf infection and eventually even trigger the formation of leaf tumor by this pathogen.

525 Therefore, to elucidate the molecular functions of these DEOs and the identification of their host 526 targets will be instrumental to gain new insights of how evolution of effector orthologs promote the 527 emergence of new pathogens.

528 With only a limited set of virulence DEOs can be tested in gene conversion experiments, this study 529 identified three effector DEOs as promising candidates for effectors with virulence function in 530 speciation. Despite these findings, one should not neglect the importance of effectors that evolved new 531 functions without fundamental changes in expression patterns. For this, the Pit2 effector is a potential 532 example: SrPit2 showed a similar expression level compared to UmPit2. However, in a previous study 533 we found that SrPit2 only partially complemented a $U$. maydis pit2 knock out mutant, which was 534 linked with a reduced ability of SrPit2 to inhibit SA-associated PLCPs (Misas Villamil et al., 2019). 535 Furthermore, a recent apoplastic proteome analysis suggested that composition of PLCPs is different 536 between maize leaf and root (Schulze Hüynck et al., 2019). Given that in natural conditions, $S$. 537 reilianum infects maize through the root while $U$. maydis infects aerial tissues, one could speculate 538 that Srpit2 adapted to be a more efficient inhibit of root PLCPs, while UmPit2 adapted to inhibit 539 PLCPs in the maize leaf. This reflects that effector function can specialize to different hosts of tissues. 540 In addition to this mechanism of gradual effector specialization, our approach to compare the 541 transcriptional levels of orthologs across two different pathogen species documented that both the 542 transcriptional plasticity and functional divergency of effector proteins contribute to pathogen 543 speciation. Including more phylogenetically related smut fungi infecting different hosts, such as $544 S$. $\square$ reilianum $f . s p$. reilianum for sorghum and $U$. hordei for barley in future research will provide a 545 more comprehensive map of the emergence and adaptation of pathogenicity effectors during host 546 adaptation and specialization. Besides the plastic regulation of effector orthogroups, one should not 547 ignore the role of species-specific effectors in virulence, which is not discussed in our study. Studies 548 on how pathogens organize their effector warehouse in temporal and spatial manner to shape the 549 pathogenesis, and how these effectors were adapted during speciation will shed a light on the 550 evolution and mechanistic basis of plant-pathogen interactions.

\section{Acknowledgments}

553 This work was funded by the European Research Council under the European Union's Horizon 2020 554 research and innovation program (consolidator grant conVIRgens, ID 771035), as well as funding by 555 the Deutsche Forschungsgemeinschaft (DFG, German Research Foundation) under Germany's 556 Excellence Strategy- EXC-2048/1- Project ID: 390686111. Weiliang Zuo and Jasper Depotter are 557 supported by the Research Fellowship Programme for Postdoctoral Researchers of the Alexander von 
558 Humboldt Foundation. MT and DKG received support by the LOEWE initiative of the government of

559 Hesse, in the framework of the LOEWE Centre for Translational Biodiversity Genomics (TBG) and

560 the LOEWE Cluster for Integrative Fungal Research (IPF).

561

562 Author contributions: W.Z. and G.D. designed the research; W.Z. performed the data analysis, 563 molecular experiment and virulence assay; J.D. and D.K.G. mapped the RNA-seq data; J.D. conducted

564 phylogenetic analysis; D.K.G. and M.T. provided ortholog expression normalization; W.Z. and G.D.

565 wrote the paper with contributions from the other authors.

566

567 Data availability

568 RNA sequencing data has been submitted to NCBI Genbank and are available under the following

569 accession: BioProject ID PRJNA674747

570

571

572

\section{References}

573

574

575

Baetsen-Young A, Wai CM, VanBuren R, Day B. 2020. Fusarium virguliforme transcriptional plasticity is revealed by host colonization of maize versus soybean. Plant Cell 32: 336-351.

576

577

Barrangou R, Fremaux C, Deveau H, Richards M, Boyaval P, Moineau S, Romero DA, Horvath P. 2007.

578 CRISPR provides acquired resistance against viruses in prokaryotes. Science.

Beckerson WC, Rodríguez De La Vega RC, Hartmann FE, Duhamel M, Giraud T, Perlin MH. 2019.

580 Cause and effectors: Whole-genome comparisons reveal shared but rapidly evolving effector sets among hostspecific plant-castrating fungi. mBio.

Begerow D, Göker M, Lutz M, Stoll M. 2004. On the evolution of smut fungi on their hosts. Frontiers in

582 basidiomycote mycology.

583 Brefort T, Tanaka S, Neidig N, Doehlemann G, Vincon V, Kahmann R. 2014. Characterization of the

584 Largest Effector Gene Cluster of Ustilago maydis. PLoS Pathogens.

585 Depotter JRL, Doehlemann G. 2020. Target the core: durable plant resistance against filamentous plant 586 pathogens through effector recognition. Pest Management Science 76: 426-431.

Depotter JRL, Zuo W, Hansen M, Zhang B, Xu M, Doehlemann G. 2020. Effectors with Different Gears: Plant Infection. Journal of Fungi 7: 16.

590 Djamei A, Schipper K, Rabe F, Ghosh A, Vincon V, Kahnt J, Osorio S, Tohge T, Fernie AR, Feussner I, et 591 al. 2011. Metabolic priming by a secreted fungal effector. Nature.

592 Doehlemann G, Van Der Linde K, ABmann D, Schwammbach D, Hof A, Mohanty A, Jackson D,

593 Kahmann R. 2009a. Pep1, a secreted effector protein of Ustilago maydis, is required for successful invasion of 594 plant cells. PLoS Pathogens.

595 Doehlemann G, Van Der Linde K, Aßmann D, Schwammbach D, Hof A, Mohanty A, Jackson D,

596 Kahmann R. 2009b. Pep1, a secreted effector protein of Ustilago maydis, is required for successful invasion of 597 plant cells. PLoS Pathogens 5.

598 Flor-Parra I, Vranes M, Kämper J, Pérez-Martín J. 2006. Biz1, a zinc finger protein required for plant 599 invasion by Ustilago maydis, regulates the levels of a mitotic cyclin. Plant Cell 18: 2369-2387. 
600 Franceschetti M, Maqbool A, Jiménez-Dalmaroni MJ, Pennington HG, Kamoun S, Banfield MJ. 2017.

601 Effectors of Filamentous Plant Pathogens: Commonalities amid Diversity. Microbiology and Molecular Biology

602 Reviews.

603 Ghareeb H, Drechsler F, Löfke C, Teichmann T, Schirawski J. 2015. SUPPRESSOR OF APICAL

604 DOMINANCE1 of Sporisorium reilianum modulates inflorescence branching architecture in maize and

605 arabidopsis. Plant Physiology.

606 Ghareeb H, Zhao Y, Schirawski J. 2019. Sporisorium reilianum possesses a pool of effector proteins that

607 modulate virulence on maize. Molecular Plant Pathology.

608 Gijzen M, Ishmael C, Shrestha SD. 2014. Epigenetic control of effectors in plant pathogens. Frontiers in Plant

609 Science.

610 Grabherr MG., Brian J. Haas, Moran Yassour Joshua Z. Levin, Dawn A. Thompson, Ido Amit, Xian 611 Adiconis, Lin Fan, Raktima Raychowdhury, Qiandong Zeng, Zehua Chen, Evan Mauceli, Nir Hacohen, 612 Andreas Gnirke, Nicholas Rhind, Federica di Palma, Bruce W. N, Friedman and AR. 2013. Trinity:

613 reconstructing a full-length transcriptome without a genome from RNA-Seq data. Nature Biotechnology 29:

$614644-652$.

615 Heimel K, Scherer M, Schuler D, Kämper J. 2010a. The Ustilago maydis Clp1 protein orchestrates

616 pheromone and b-dependent signaling pathways to coordinate the cell cycle and pathogenic development. Plant

617 Cell.

618 Heimel K, Scherer M, Vranes M, Wahl R, Pothiratana C, Schuler D, Vincon V, Finkernagel F, Flor-Parra I, Kämper J. 2010b. The transcription factor rbf1 is the master regulator for b-mating type controlled pathogenic development in Ustilago maydis. PLoS Pathogens.

621 Hemetsberger C, Herrberger C, Zechmann B, Hillmer M, Doehlemann G. 2012. The Ustilago maydis

622 effector Pep1 suppresses plant immunity by inhibition of host peroxidase activity. PLoS Pathogens 8.

623 Hemetsberger C, Mueller AN, Matei A, Herrberger C, Hensel G, Kumlehn J, Mishra B, Sharma R,

624 Thines M, Hückelhoven R, et al. 2015. The fungal core effector Pep1 is conserved across smuts of dicots and

625 monocots. New Phytologist.

626 Irieda H, Inoue Y, Mori M, Yamada K, Oshikawa Y, Saitoh H, Uemura A, Terauchi R, Kitakura S,

627 Kosaka A, et al. 2019. Conserved fungal effector suppresses PAMP-triggered immunity by targeting plant

628 immune kinases. Proceedings of the National Academy of Sciences of the United States of America.

629 Jones JDG, Dangl JL. 2006. The plant immune system. Nature 444: 323-329.

630 Kämper J, Kahmann R, Bölker M, Ma LJ, Brefort T, Saville BJ, Banuett F, Kronstad JW, Gold SE,

631 Müller O, et al. 2006a. Insights from the genome of the biotrophic fungal plant pathogen Ustilago maydis.

632 Nature.

633 Kämper J, Kahmann R, Bölker M, Ma LJ, Brefort T, Saville BJ, Banuett F, Kronstad JW, Gold SE, 634 Müller O, et al. 2006b. Insights from the genome of the biotrophic fungal plant pathogen Ustilago maydis. 635 Nature 444: 97-101.

636 Katoh K, Standley DM. 2013. MAFFT multiple sequence alignment software version 7: Improvements in 637 performance and usability. Molecular Biology and Evolution 30: 772-780.

638 Langmead B, Salzberg SL. 2012. Fast gapped-read alignment with Bowtie 2. Nature Methods 9: 357-359.

639 Lanver D, Berndt P, Tollot M, Naik V, Vranes M, Warmann T, Münch K, Rössel N, Kahmann R. 2014.

640 Plant Surface Cues Prime Ustilago maydis for Biotrophic Development. PLoS Pathogens.

641 Lanver D, Müller AN, Happel P, Schweizer G, Haas FB, Franitza M, Pellegrin C, Reissmann S, Altmüller

642 J, Rensing SA, et al. 2018. The biotrophic development of ustilago maydis studied by RNA-seq analysis. Plant

643 Cell.

644 Lanver D, Tollot M, Schweizer G, Lo Presti L, Reissmann S, Ma LS, Schuster M, Tanaka S, Liang L,

645 Ludwig N, et al. 2017. Ustilago maydis effectors and their impact on virulence. Nature Reviews Microbiology. 
646

647

648

649

650

651

652

653

654

655

656

657

658

659

660

661

662

663

664

665

666

667

668

669

670

671

672

673

674

675

676

677

678

679

680

681

682

683

684

685

686

687

688

689

690

Laurie JD, Ali S, Linning R, Mannhaupt G, Wong P, Güldener U, Münsterkötter M, Moore R, Kahmann R, Bakkeren G, et al. 2012. Genome comparison of barley and maize smut fungi reveals targeted loss of RNA silencing components and species-specific presence of transposable elements. Plant Cell.

Liao Y, Smyth GK, Shi W. 2019. The R package Rsubread is easier, faster, cheaper and better for alignment and quantification of RNA sequencing reads. Nucleic Acids Research $\mathbf{4 7}$.

Ma LS, Wang L, Trippel C, Mendoza-Mendoza A, Ullmann S, Moretti M, Carsten A, Kahnt J, Reissmann S, Zechmann B, et al. 2018. The Ustilago maydis repetitive effector Rsp3 blocks the antifungal activity of mannose-binding maize proteins. Nature Communications.

Martinez C, Roux C, Jauneau A, Dargent R. 2002. The biological cycle of Sporisorium reilianum f.sp. zeae: An overview using microscopy. Mycologia 94: 505-514.

Matei A, Ernst C, Günl M, Thiele B, Altmüller J, Walbot V, Usadel B, Doehlemann G. 2018. How to make a tumour: cell type specific dissection of Ustilago maydis-induced tumour development in maize leaves. New Phytologist.

Misas Villamil JC, Mueller AN, Demir F, Meyer U, Ökmen B, Schulze Hüynck J, Breuer M, Dauben H, Win J, Huesgen PF, et al. 2019. A fungal substrate mimicking molecule suppresses plant immunity via an interkingdom conserved motif. Nature Communications $\mathbf{1 0 .}$

Morita T, Koike H, Koyama Y, Hagiwara H, Ito E, Fukuoka T, Imura T, Machida M, Kitamoto D. 2013. Genome sequence of the basidiomycetous yeast Pseudozyma antarctica T-34, a producer of the glycolipid biosurfactants mannosylerythritol lipids. Genome Announcements.

Mueller AN, Ziemann S, Treitschke S, Aßmann D, Doehlemann G. 2013. Compatibility in the Ustilago maydis-Maize Interaction Requires Inhibition of Host Cysteine Proteases by the Fungal Effector Pit2. PLoS Pathogens 9.

Raffaele S, Kamoun S. 2012. Genome evolution in filamentous plant pathogens: Why bigger can be better. Nature Reviews Microbiology 10: 417-430.

Redkar A, Hoser R, Schilling L, Zechmann B, Krzymowska M, Walbot V, Doehlemann G. 2015a. A secreted effector protein of Ustilago maydis guides maize leaf cells to form tumors. Plant Cell.

Redkar A, Villajuana-Bonequi M, Doehlemann G. 2015b. Conservation of the Ustilago maydis effector see1 in related smuts. Plant Signaling and Behavior.

Robinson MD, McCarthy DJ, Smyth GK. 2009. edgeR: A Bioconductor package for differential expression analysis of digital gene expression data. Bioinformatics.

Sánchez-Vallet A, Fouché S, Fudal I, Hartmann FE, Soyer JL, Tellier A, Croll D. 2018. The genome biology of effector gene evolution in filamentous plant pathogens. Annual Review of Phytopathology 56: 21-40.

Schilling L, Matei A, Redkar A, Walbot V, Doehlemann G. 2014. Virulence of the maize smut Ustilago maydis is shaped by organ-specific effectors. Molecular Plant Pathology.

Schirawski J, Mannhaupt G, Münch K, Brefort T, Schipper K, Doehlemann G, Di Stasio M, Rössel N, Mendoza-Mendoza A, Pester D, et al. 2010. Pathogenicity determinants in smut fungi revealed by genome comparison. Science.

Schnable PS, Ware D, Fulton RS, Stein JC, Wei F, Pasternak S, Liang C, Zhang J, Fulton L, Graves TA, et al. 2009. The B73 maize genome: Complexity, diversity, and dynamics. Science 326.

Schulze Hüynck J, Kaschani F, van der Linde K, Ziemann S, Müller AN, Colby T, Kaiser M, Misas Villamil JC, Doehlemann G. 2019. Proteases underground: Analysis of the maize root apoplast identifies organ specific papain-like cysteine protease activity. Frontiers in Plant Science.

Schuster M, Kahmann R. 2019. CRISPR-Cas9 genome editing approaches in filamentous fungi and oomycetes. Fungal Genetics and Biology.

Schuster M, Schweizer G, Kahmann R. 2018. Comparative analyses of secreted proteins in plant pathogenic 
smut fungi and related basidiomycetes. Fungal Genetics and Biology.

692 Schuster M, Schweizer G, Reissmann S, Kahmann R. 2016. Genome editing in Ustilago maydis using the

693 CRISPR-Cas system. Fungal Genetics and Biology.

694 Schweizer G, Münch K, Mannhaupt G, Schirawski J, Kahmann R, Dutheil JY. 2018. Positively Selected Effector Genes and Their Contribution to Virulence in the Smut Fungus Sporisorium reilianum. Genome Biology

696 and Evolution.

697 Selin C, de Kievit TR, Belmonte MF, Fernando WGD. 2016. Elucidating the role of effectors in plant-fungal interactions: Progress and challenges. Frontiers in Microbiology 7: 1-21.

699 Seppey M, Manni M, Zdobnov EM. 2019. BUSCO: Assessing genome assembly and annotation completeness.

700 In: Methods in Molecular Biology.

701 Sharma R, Mishra B, Runge F, Thines M. 2014. Gene Loss rather than gene gain is associated with a host

702 jump from monocots to dicots in the smut fungus melanopsichium pennsylvanicum. Genome Biology and

703 Evolution.

704 Skibbe DS, Doehlemann G, Fernandes J, Walbot V. 2010. Maize tumors caused by ustilago maydis require

705 organ-specific genes in host and pathogen. Science.

706 Stamatakis A. 2014. RAxML version 8: A tool for phylogenetic analysis and post-analysis of large phylogenies.

707 Bioinformatics 30: 1312-1313.

708 Stirnberg A, Djamei A. 2016. Characterization of ApB73, a virulence factor important for colonization of Zea

709 mays by the smut Ustilago maydis. Molecular Plant Pathology.

710 Sucher J, Mbengue M, Dresen A, Barascud M, Didelon M, Barbacci A, Raffaele S. 2020.

711 Phylotranscriptomics of the pentapetalae reveals frequent regulatory variation in plant local responses to the

712 fungal pathogen sclerotinia sclerotiorum. Plant Cell 32: 1820-1844.

713 Tanaka S, Brefort T, Neidig N, Djamei A, Kahnt J, Vermerris W, Koenig S, Feussner K, Feussner I,

714 Kahmann R. 2014. A secreted Ustilago maydis effector promotes virulence by targeting anthocyanin

715 biosynthesis in maize. eLife.

716 Tanaka S, Schweizer G, Rössel N, Fukada F, Thines M, Kahmann R. 2019. Neofunctionalization of the

717 secreted Tin2 effector in the fungal pathogen Ustilago maydis. Nature Microbiology.

718 Taniguti LM, Schaker PDC, Benevenuto J, Peters LP, Carvalho G, Palhares A, Quecine MC, Nunes FRS,

719 Kmit MCP, Wai A, et al. 2015. Complete genome sequence of Sporisorium scitamineum and biotrophic

720 interaction transcriptome with sugarcane. PLOS ONE.

Thines M. 2019. An evolutionary framework for host shifts - jumping ships for survival. New Phytologist 224: 605-617.

Tollot M, Assmann D, Becker C, Altmüller J, Dutheil JY, Wegner CE, Kahmann R. 2016. The WOPR

Protein Ros1 Is a Master Regulator of Sporogenesis and Late Effector Gene Expression in the Maize Pathogen Ustilago maydis. PLoS Pathogens 12.

Torres DE, Oggenfuss U, Croll D, Seidl MF. 2020. Genome evolution in fungal plant pathogens: looking beyond the two-speed genome model. Fungal Biology Reviews.

Toruño TY, Stergiopoulos I, Coaker G. 2016. Plant-Pathogen Effectors: Cellular Probes Interfering with Plant Defenses in Spatial and Temporal Manners. Annual Review of Phytopathology 54: 419-441.

Zahiri A, Heimel K, Wahl R, Rath M, Kämper J. 2010. The Ustilago maydis forkhead transcription factor Fox 1 is involved in the regulation of genes required for the attenuation of plant defenses during pathogenic

732 development. Molecular Plant-Microbe Interactions.

733 Zuo W, Depotter JR, Doehlemann G. 2020. Cas9HF1 enhanced specificity in Ustilago maydis. Fungal

734 Biology 124: 228-234. 


\section{Figure Legends}

Fig.1. Comparison of $U$. maydis and $S$. reilianum growth during leaf infection. a, calcofluor white staining of fungal hyphae and appressorium at 20hpi. b-d, WGA-AF488Propidium Iodide co-staining to show the fungal biotrophic growth inside the plant cell. Fungal cells were stained with WGA-AF488 (green), the vascular cell wall was stained with Propidium Iodide (red). White arrows indicate the de-novo divided bundle sheath cells triggered by $U$. maydis infection. White circles indicate the aggregation of $S$. reilianum cells inside the leaf vein. e, the relative biomass quantification by qPCR shows the fungal growth during infection, and a significant higher abundance of $S$. reilianum was detected at 4 dpi. $\mathbf{f}$, the relative fungal reads from RNA-seq, which was the ratio between reads uniquely mapped to fungi and total reads uniquely mapped (fungi and maize). A significantly higher number of mapped reads was detected from $S$. reilianum at 4 dpi. “**”, Experiments have been performed in three independent biological replicates. $p<0.05$. Student $t$-test was used for significance test.

Fig.2. Overview of $U$. maydis and $S$. reilianum transcriptome, respectively. a, PCA analysis of $U$. maydis and $S$. reilianum RNA-seq samples. b, expression data from three different growth conditions. The number of differentially expressed genes ( $\log 2$ fold change $>1, \mathrm{p}>0.05$ ) from pairwise comparison were indicated in the triangles. Red and green triangles represent up-regulated and down-regulated genes between 2 dpi vs. axenic culture (AC), 4 dpi vs. AC and 4 dpi vs. 2 dpi, respectively. c, boxplot shows transcriptional changes of effector and non-effector genes during biotrophic growth. Effector genes were dramatically up-regulated compared to the non-effector genes. d, heatmap shows the overview of the expression changes of effector genes. "n" shows the number of effectors detected expressing in RNA-seq sample and the total number of effectors in the genome.

Fig.3. The induced pattern shows the expression changes of effectors. a, the induced effectors are clustered into 5 patterns based on the expression changes between axenic culture (AC), 2 dpi and 4 dpi conditions. The gray lines represent individual gene, and blue and orange lines are the mean of all genes in each cluster. "a", "b" and "c" were used to indicate the significance levels between each condition. Genes with $\log 2$ fold change $>1$ and $p<0.05$ were considered significantly induced in pairwise comparison. $\mathbf{b}$, the number of induced genes of each pattern. The chi-square test was used for statistic test for the significance. " ***”, $p<0.01$.

Fig.4. Orthologs were differentially regulated between $U$. maydis and $S$. reilianum. a, PCA analysis based on the relative ortholog expression of 6005 one-to-one ortholog pairs from $U$. maydis and $S$. reilianum. $\mathbf{b}$, the number of DEOs detected in different conditions. The fold change $>3$ and $\mathrm{p}<0.05$ were used to identify DEO. c, the GO classification of DEOs in three different conditions. d, the Venn diagram shows the number of differentially expressed effector orthologs during biotrophic growth. e-f, the differentially regulation of effectors clustered by the conservation between 5 smut fungi. e, the phylogenetic tree of 5 smut fungi, including U. maydis, S. reilianum, Ustilago hordei, S. scitamineum and M. pennsylvanicum. f, the number of effector DEOs in each category. Chi-square test was used for significance test. “**", $p<0.01$, “****, $p<0.001$.

Fig.5. Effector orthologs between $U$. maydis and $S$. reilianum are functional conserved. a, a heatmap shows the expression difference and protein sequence identity of effectors ortholog pairs which have been knocked out and subjected to virulence tests in U. maydis. "đI", Pit2 only conserved in functional PID motif which failed to be detected during ortholog 
787 identification and "\%ID" was not showed. b, the scheme demonstrated the process of 788 CRISPR-Cas9 mediated gene conversion. The sgRNA was designed to target the coding 789 sequence of $U$. maydis effector, and the donor plasmid contains $S$. reilianum ortholog coding 790 sequence flanked by $1 \mathrm{~kb}$ homologous region. c-d, Disease scoring results of gene conversion 791 mutants and corresponding U. maydis knock out mutant. c, Converted UMAG_05306 and 792 UMAG_11060 to the S. reilianum orthologs did not affect the virulence in SG200. d,

\section{Supplementary Captions}

Supplementary Fig.1 The symptom of $U$. maydis and $S$. reilianum infected leaves. a, The photos shows the symptom development from 2 dpi to $4 \mathrm{dpi}$. The dashed circle shows the first visible tumor from $U$. maydis infected leaf while the dashed rectangle shows the necrosis spot caused by $S$. reilianum infection. The back of $U$. maydis infected leaf from 4 dpi is shown to better visualize the tumor. b, Confocal microscopy shows the details of the $S$. reilianum aggregation inside the leaf vascular at 4 dpi after WGA-AF488- Propidium Iodide staining. The fungal cells aggregate inside the vascular tissue. Scale bar=100 $\mu \mathrm{m}$.

Supplementary Fig.2 qPCR confirms the expression of effector genes from $\boldsymbol{U}$. maydis and $S$. reilianum. a, Expression levels of $U$. maydis effector genes. b, Expression levels of $S$. reilianum effector genes. Infection markers such as UmPepl and UmPit2 and their corresponding orthologs were detected, as well as organ-specific effector See1 and two differentially expressed orthogroups UMAG_05306/Sr10059 and UMAG_11060/Sr14941. The relative expression levels of effectors were normalized by respective reference peptidylprolyl isomerase (ppi) genes by $2^{-\Delta \mathrm{Ct}}$ method. Error bar indicate standard deviation from three biological replications.

Supplementary Fig.3 Differential regulation of orthologs between $U$. maydis and $S$. reilianum from different samples. a, Pearson correlation analysis between $U$. maydis and $S$. reilianum samples based on relative ortholog expression from 6005 one-to-one orthologs. The $r$ values indicate a high to low correlation between $U$. maydis and $S$. reilianum samples: AC>2 dpi $>4$ dpi. b, Venn diagram shows the number of differentially expressed ortholog in $U$. maydis and S. reilianum from different conditions. About $30 \%$ and $50 \%$ of detected DEOs were only detected from axenic culture.

Supplementary Fig.4 Expression of pathogenic development related transcription factors between $U$. maydis and $S$. reilianum during biotrophic growth from RNA-seq data. The transcription factors $R b f 1$ and Bizl were highly expressed in S. reilianum while transcription factors $H d p 2$, Foxl and Nltl were enhanced expressed in $U$. maydis. The transcription factor Ros 1 shows similar expression between these two smut fungi. Student $t$ test was used for significance test. “**”, $p<0.01$.

Supplementary Fig.5 The distribution of effector DEOs on chromosomes. a, the distribution of DEOs in 22 chromosomes based on the $U$. maydis genome organization. The blue bar shows $U$. maydis highly expressed DEOs, and the yellow bar shows the $S$. reilianum highly expressed DEOs. 2 dpi DEOs and 4 dpi DEOs were plotted above and below the 
838 chromosome, respectively. The red rectangle shows effector cluster 19A. b, the diagram 839 shows the overview of synteny of effectors in cluster 19A. The effector genes are showed in 840 green arrows; non-effector genes are shown in dark gray. The synteny of effector genes are in 841 grey shade, the outline color of the shade indicates the DEO. The U. maydis highly expressed 842 effector ortholog pairs are outlined in blue, the $S$. reilianum highly expressed genes are 843 outlined in yellow.

851 Supplementary Fig. 7 The regulation of $S$. reilianum promoters in $U$. maydis by 852 CRIPSR-Cas9 mediated promoter conversion. a, Schematic model of the method used for 853 CRISPR-Cas9 mediated promoter conversion. b, qPCR detection of the corresponding 854 effectors expression under different orthologous $S$. reilianum promoters. At 4 dpi, Pro ${ }^{\text {sr10059 }}$ 855 regulates gene expression to the similar level as Pro ${ }^{\mathrm{UMAG}-05306}$ in $U$. maydis, while at 2 dpi, 856 Pro ${ }^{\text {sr14941 }}$ drove gene expression was significantly lower compared to Pro ${ }^{\text {UMAG_11060 }}$. Error bar 857 indicates standard deviation from three biological replications.

858

859 Supplement Table.1 Oligo sequences used.

860 Supplement Table.2 Log2 CPM (count per million) of fungal reads.

861 Supplement Table.3 Relative ortholog expression data of 6005 one-to-one orthologs 862 between $\boldsymbol{U}$. maydis and S. reilianum. 
bioRxiv preprint doi: https://doi.org/10.1101/2020.11.03.366443; this version posted May 7, 2021. The copyright holder for this preprint (which was not certified by peer review) is the author/funder. All rights reserved. No reuse allowed without permission.

Figure 1
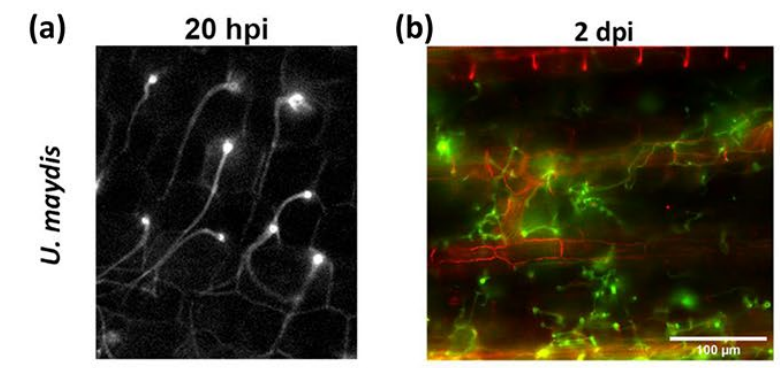

(c)

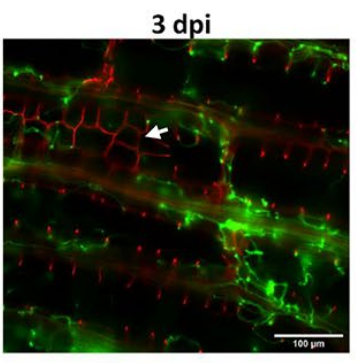

(d)
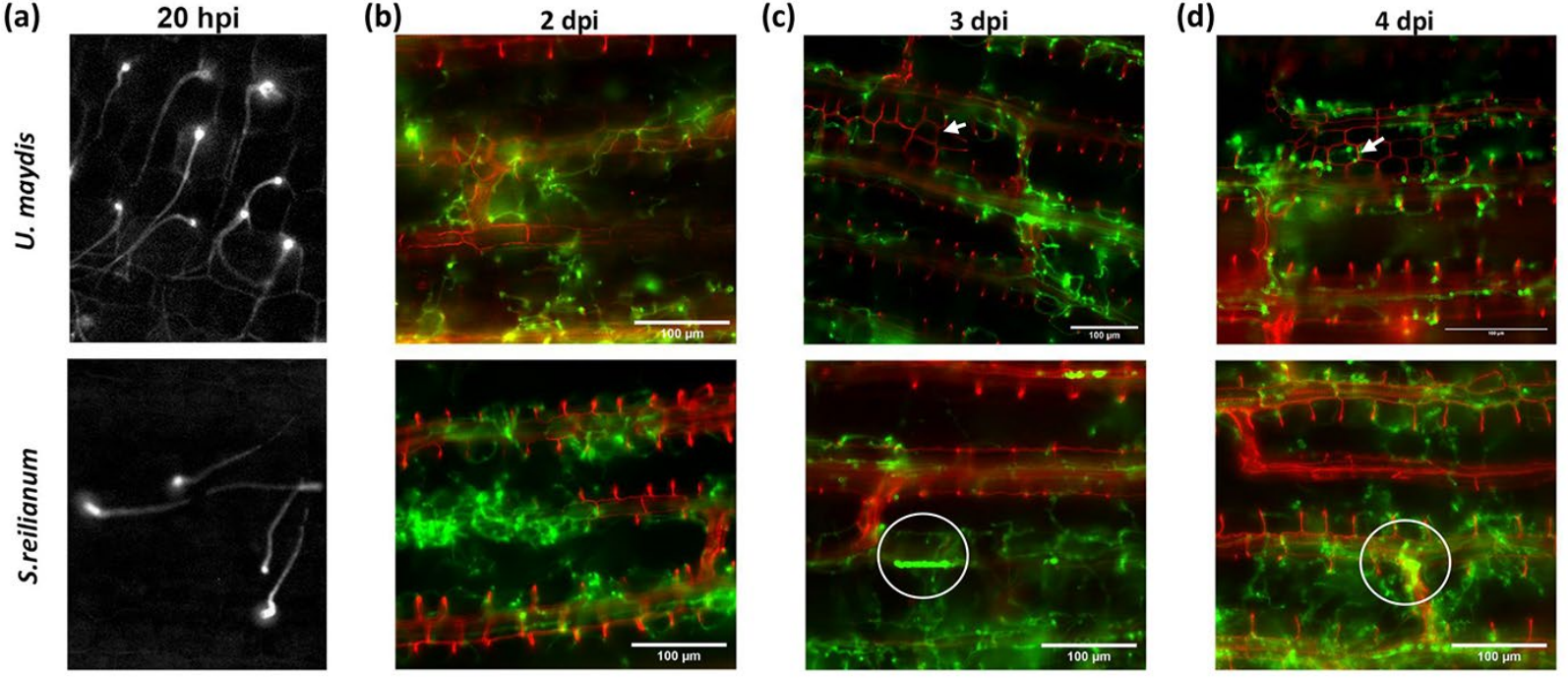

(e)

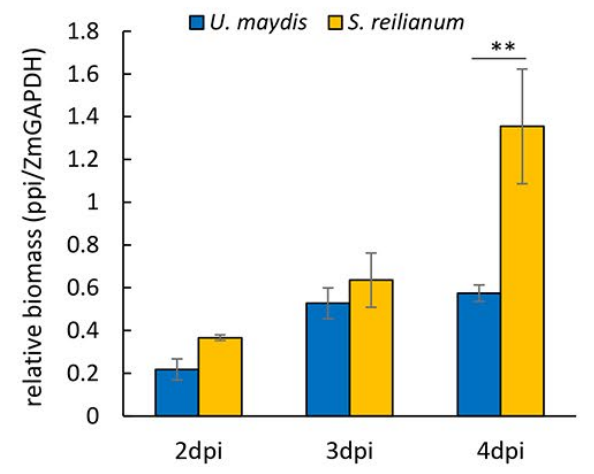

(f)




bioRxiv preprint doi: https://doi.org/10.1101/2020.11.03.366443; this version posted May 7, 2021. The copyright holder for this preprint (which was not certified by peer review) is the author/funder. All rights reserved. No reuse allowed without permission.

\section{Figure 2}

(a)

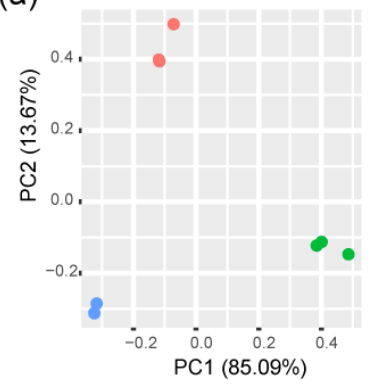

(c)
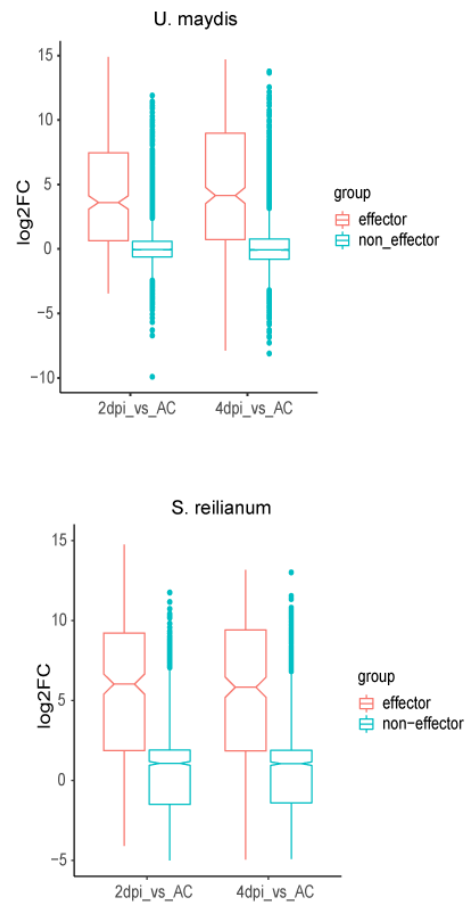

(b)
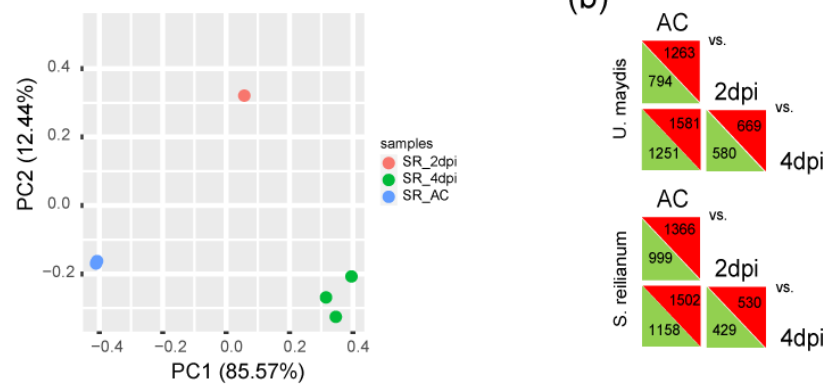

(d)

bioRxiv preprint doi: https://doi.org/10.1101/2020.11.03.366443; this version posted May 7, 2021. The copyright holder for this preprint (which was not certified by peer review) is the author/funder. All rights reserved. No reuse allowed without permission.

\section{Figure 3}

(a)
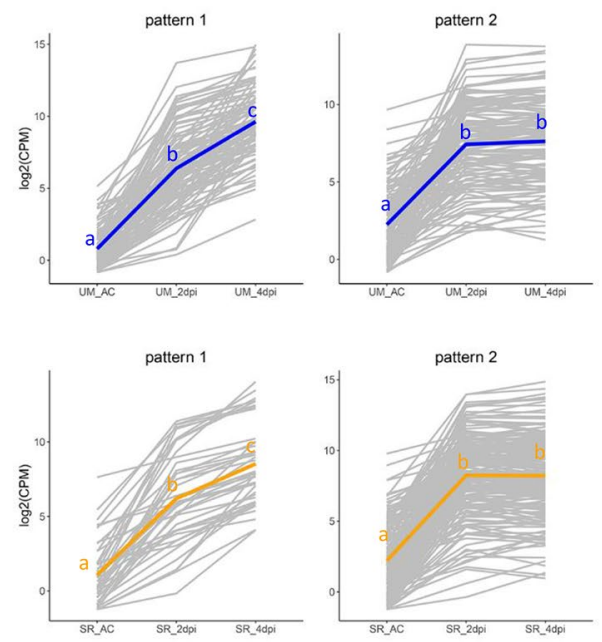

(b)
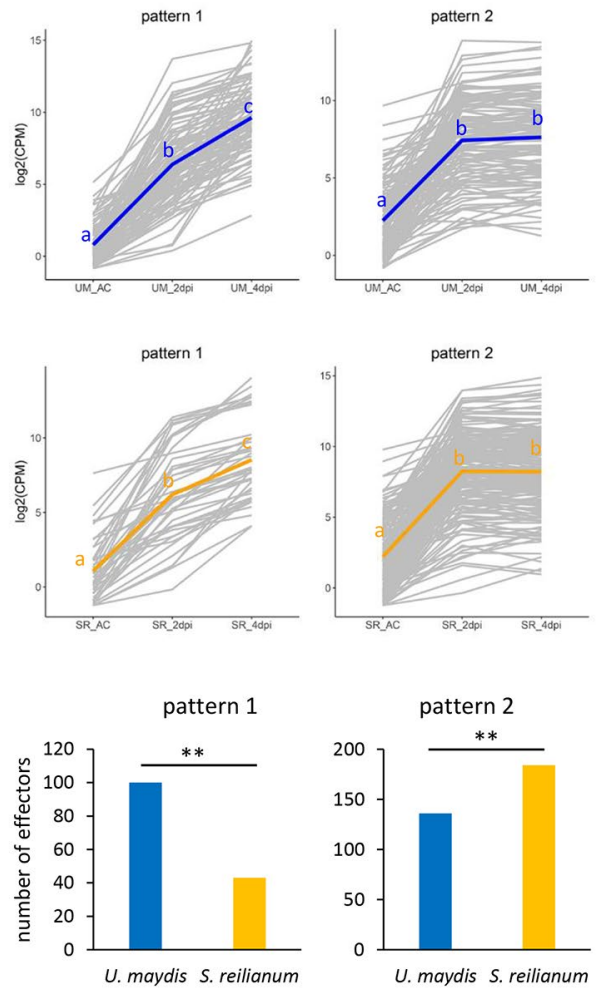
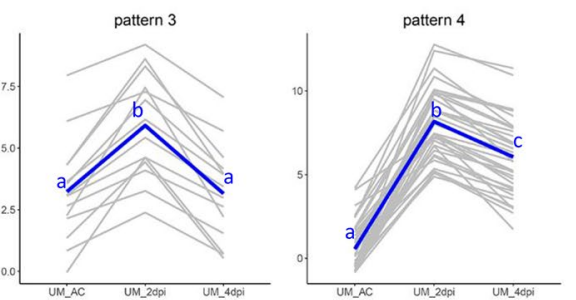

pattern 3
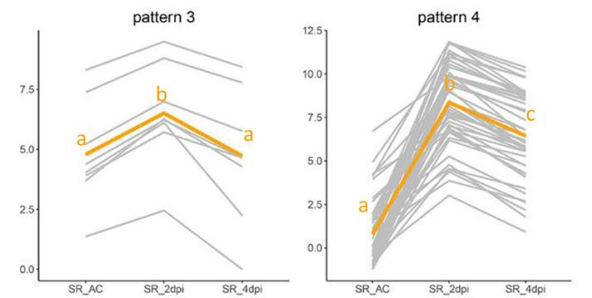

pattern 3

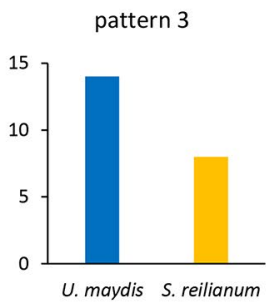

pattern 4

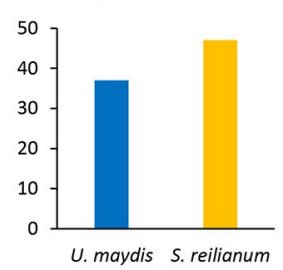

pattern 5



pattern 5



pattern 5

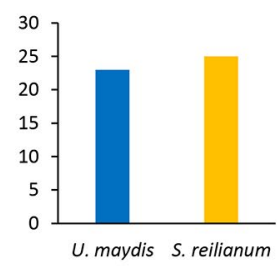


bioRxiv preprint doi: https://doi.org/10.1101/2020.11.03.366443; this version posted May 7, 2021. The copyright holder for this preprint (which was not certified by peer review) is the author/funder. All rights reserved. No reuse allowed without permission.

\section{Figure 4}

(a)

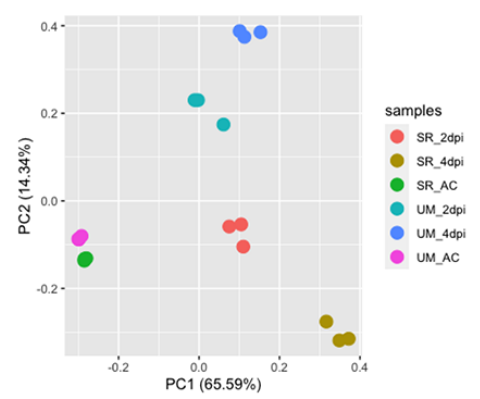

(b)

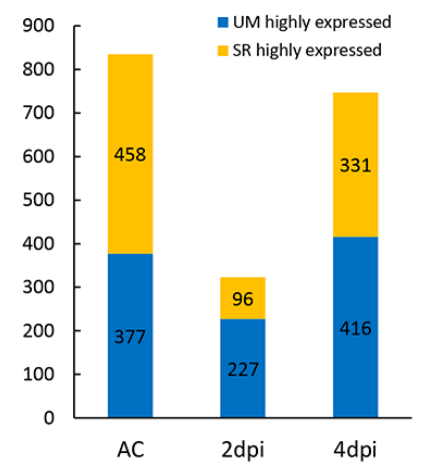

(d)

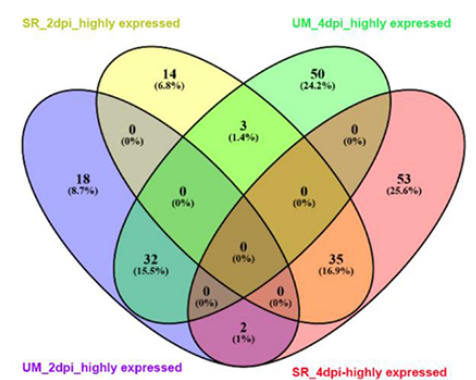

(c)

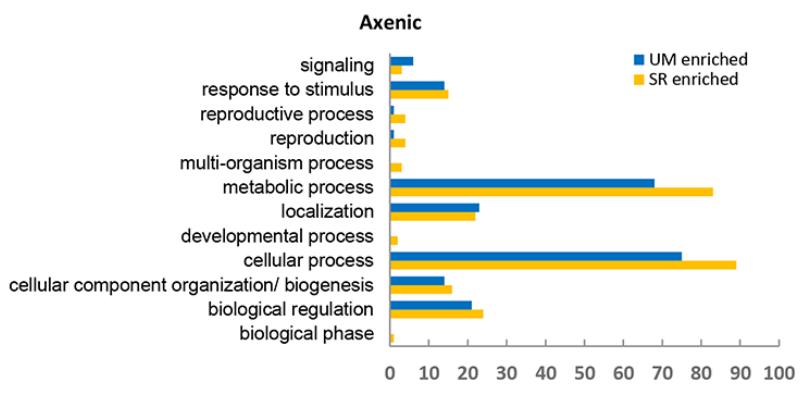

2dpi

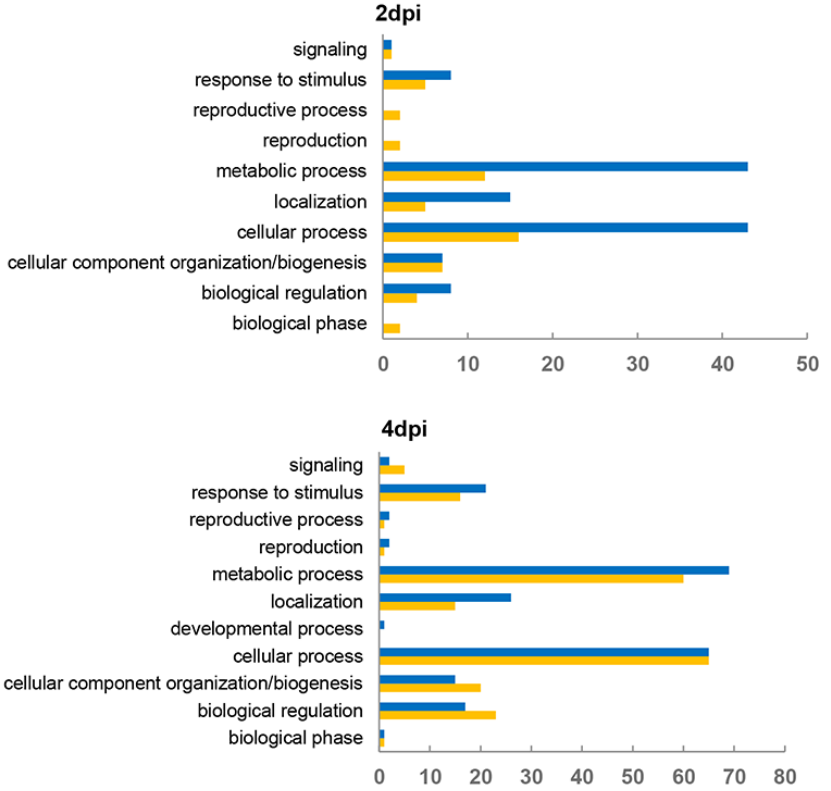

(e)

0.05




bioRxiv preprint doi: https://doi.org/10.1101/2020.11.03.366443; this version posted May 7, 2021. The copyright holder for this preprint (which was not certified by peer review) is the author/funder. All rights reserved. No reuse allowed without permission.

Figure 5
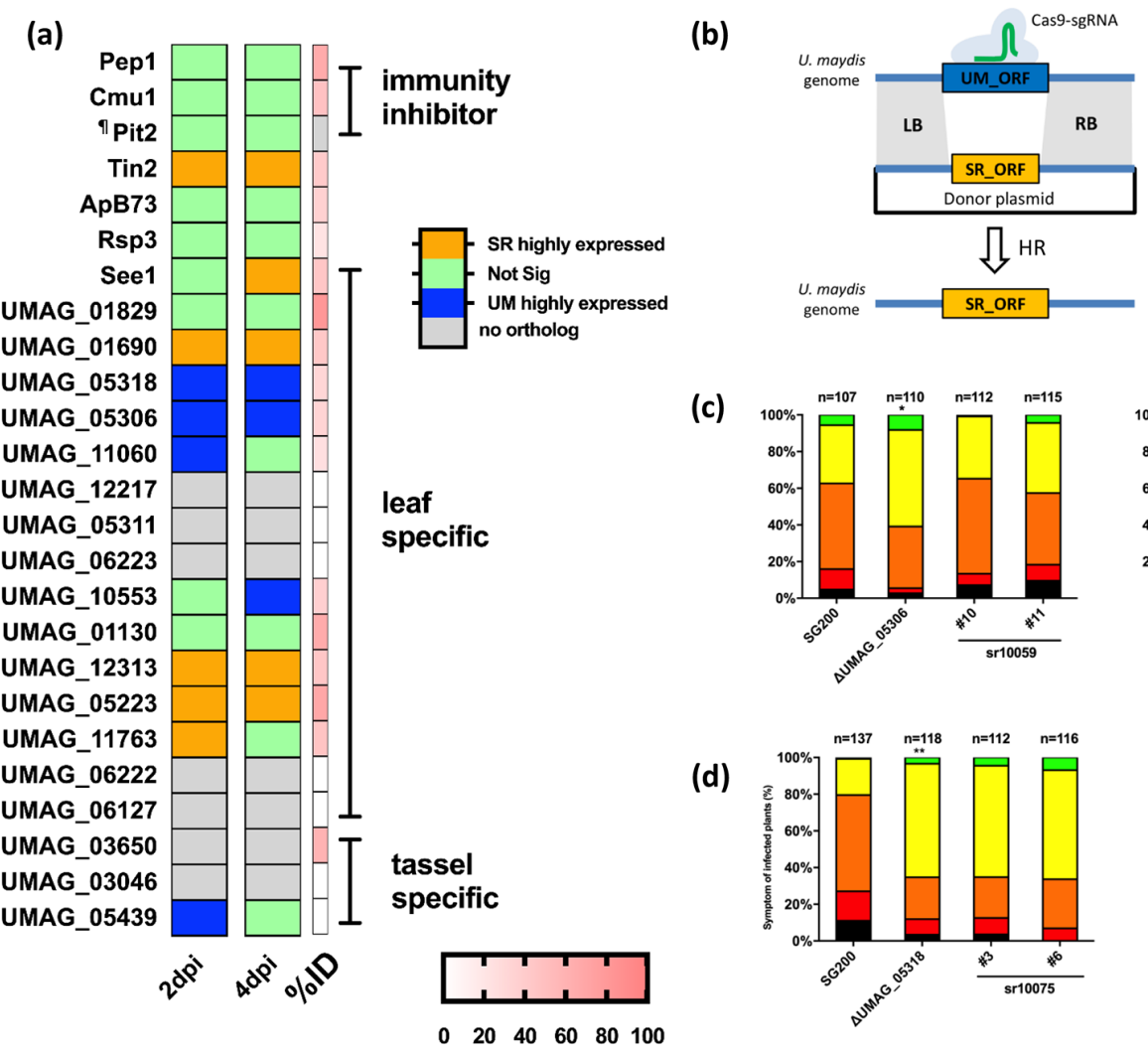

(c)
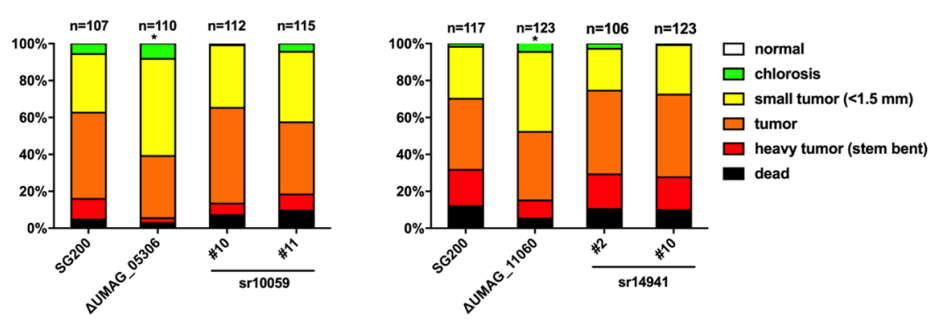

(d)
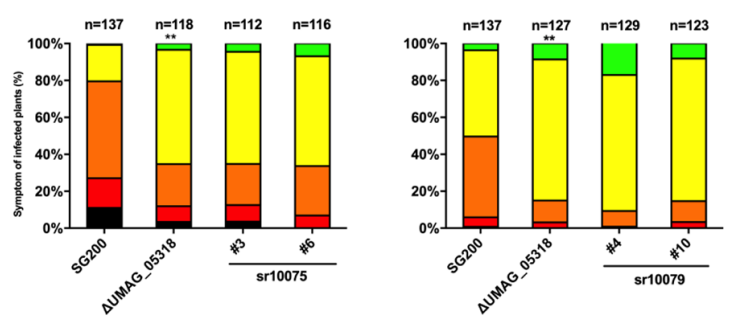